\title{
Qualidade de frutos de melancia sob diferentes dosagens de adubação nitrogenada
}

\author{
Quality of watermelon fruits under different doses of nitrogen fertilization
}

Calidad de frutos de sandía bajo diferentes dosis de fertilización nitrogenada

\section{Resumo}

Este trabalho teve como intuito analisar o efeito da aplicação de nitrogênio sobre os componentes de qualidade de frutos da melancia "granada". Para isso, foi realizado um experimento na fazenda Extrema, situada no município de Arinos, estado de Minas Gerais, no período de setembro a dezembro de 2018. O delineamento experimental adotado foi o de blocos inteiramente casualizados, com cinco tratamentos e quatro repetições. Os tratamentos constituíram-se de cinco níveis de adubação nitrogenada: 0, 40, 80, 160 e $320 \mathrm{~kg} \mathrm{ha}^{-1} \mathrm{de} \mathrm{N}$. As análises dos frutos foram feitas no laboratório de Química do Instituto Federal de Educação, Ciência e Tecnologia Norte de Minas Gerais - Campus Arinos. Foram realizados testes físico-químicos para a determinação de sólidos solúveis totais $\left({ }^{\circ} \mathrm{Brix}\right)$, pH, acidez titulável (AT) e espessura de casca. A adubação nitrogenada apresentou efeito significativo sobre as variáveis analisadas, exceto no $\mathrm{pH}$ dos frutos. O maior teor de sólidos solúveis $\left(8,27^{\circ}\right.$ Brix) foi obtido ao nível de $171,7 \mathrm{~kg}^{\circ}{ }^{-1}$ de N. Para AT e espessura de casca, foram ajustados modelos lineares significativos $(\mathrm{p}<0,01)$.

Palavras-chave: Cucurbitácea; Sólidos solúveis; Acidez titulável; Nitrogênio.

\begin{abstract}
The objective of this work was to analyze the effect of nitrogen application on the fruit quality components of "granada" watermelon. For this, an experiment was carried out at Farm Extrema, located in the municipality of Arinos, state of Minas Gerais, from September to December 2018. The experimental design was a completely randomized block with five treatments and four replications. The treatments consisted of five levels of nitrogen fertilization: $0,40,80,160$ and $320 \mathrm{~kg} \mathrm{ha}^{-1}$ of $\mathrm{N}$. Fruit analyzes were carried out at the Chemistry laboratory of the Federal Institute of Education, Science and Technology of Minas Gerais - Campus Arinos. Physicochemical tests were performed to determine total soluble solids ( ${ }^{\circ}$ Brix), $\mathrm{pH}$, titratable acidity (TA) and shell thickness. Nitrogen fertilization had a significant effect on the analyzed variables, except the $\mathrm{pH}$ of the fruits. The highest soluble solids content $\left(8.27^{\circ}\right.$ Brix) was obtained at the level of $171.7 \mathrm{~kg} \mathrm{ha}^{-1}$ of $\mathrm{N}$. For TA and bark thickness, significant linear models were adjusted $(\mathrm{p}<0.01)$.
\end{abstract}

Keywords: Cucurbits; Soluble solids; Titratable acidity; Nitrogen.

\section{Resumen}

Este trabajo tuvo como objetivo analizar el efecto de la aplicación de nitrógeno en los componentes de calidad de frutos de sandía "granada". Para ello, se realizó un experimento en la hacienda Extrema, ubicada en el municipio de 
Arinos, estado de Minas Gerais, de septiembre a diciembre de 2018. El diseño experimental adoptado fue de bloques completamente al azar, con cinco tratamientos y cuatro repeticiones. Los tratamientos consistieron en cinco niveles de fertilización nitrogenada: 0, 40, 80, 160 y $320 \mathrm{~kg} \mathrm{ha}^{-1}$ de $\mathrm{N}$. Los análisis de los frutos se realizaron en el Laboratorio de Química del Instituto Federal de Educación, Ciencia y Tecnología Norte de Minas Gerais - Campus Arinos. Se realizaron pruebas físico-químicas para determinar sólidos solubles totales $\left({ }^{\circ} \mathrm{Brix}\right), \mathrm{pH}$, acidez titulable $(\mathrm{AT})$ y espesor de la cáscara. La fertilización nitrogenada tuvo un efecto significativo en las variables analizadas, excepto en el $\mathrm{pH}$ de los frutos. La fertilización nitrogenada tuvo un efecto significativo en las variables analizadas, excepto en el $\mathrm{pH}$ de los frutos. El mayor contenido de sólidos solubles $\left(8,27^{\circ}\right.$ Brix) se obtuvo al nivel de $171,7 \mathrm{~kg} \mathrm{ha}^{-1}$ de $\mathrm{N}$. Para AT y espesor de corteza se ajustaron modelos lineales significativos $(\mathrm{p}<0,01)$.

Palabras clave: Cucurbitáceas; Sólidos solubles; Acidez valorable; Nitrógeno.

\section{Introdução}

A melancia é uma das principais espécies olerícolas cultivadas no Brasil, em especial na região Nordeste, que de acordo com o IBGE (2012) respondeu por 34,2\% da produção do país, com destaque para os estados de Pernambuco e da Bahia, que juntos foram responsáveis por $63 \%$ da produção regional. Isso se deve as condições de solo e clima favoráveis ao seu desenvolvimento, podendo ser cultivada o ano inteiro sob condições irrigadas. A região noroeste de Minas Gerais apresenta condições edafoclimáticas semelhantes aos da região nordeste, o que faz a região ter, também, potencial produtivo desta olerícola.

No Brasil, as médias anuais de produção e de área cultivada foram de 2.314 .700 toneladas e 105.491 hectares em 2017. O Estado do Rio Grande do Sul é o maior produtor, com 346.310 toneladas, seguido pelo Estado de São Paulo, com 291.846 toneladas, e Goiás, com 267.896 toneladas. Já o estado de Minas Gerais alcançou uma produção de 23.947 toneladas nesse mesmo ano (IBGE, 2017). As exportações estão em declínio, após pico de vendas em setembro/2018. Em janeiro/2018, o quantitativo foi de 7,74 mil toneladas, número 6,98\% menor em relação ao mesmo mês do ano passado e também menor em relação a dezembro de 2017, na ordem de 31,98\%. O valor da comercialização foi de US\$ 4,4 milhões, superior 12,58\% em relação ao mesmo período do ano anterior. A Europa continua como principal destino da fruta tropical, boa opção frente a oscilações no mercado interno. Entretanto, apesar dos bons embarques até o momento, por causa de problemas climáticos que afetam a produtividade as exportações podem ser afetadas nos próximos meses (CONAB, 2018).

De importante valor nutritivo, o consumo de melancia não é maior devido ao grande tamanho dos frutos e à dificuldade no descascamento, tornando seu preparo muito trabalhoso (Miguel et al., 2007). Além do tamanho, outros aspectos são igualmente importantes e devem ser relacionados ao desenvolvimento do fruto para realização da colheita, como polpa vermelha, o teor de sólidos solúveis acima de $8 \%$, o formato arredondado do fruto e a resistência a doenças (Ferreira et al., 2003).

Quanto ao desenvolvimento da melancieira, vários fatores referentes ao solo, ao clima e a planta possuem interferência direta no rendimento da cultura. Dentre esses fatores, a água e o nitrogênio (N) merecem destaque especial não só pelo custo de produção que, juntos, representam cerca de $10 \%$ no caso da melancia, mas também devido à necessidade de se utilizar a água e o nitrogênio eficientemente de modo a garantir a disponibilidade da água e manter o solo com condições de ser utilizado por gerações futuras. Alia-se a essas considerações o fato destes dois recursos proporcionarem as maiores variações no rendimento da cultura, em função dos níveis utilizados. A exploração ótima do ponto de vista econômico de uma cultura requer a utilização de níveis adequados destes fatores (Hexem \& Heady, 1978).

Já os componentes de produção e de qualidade dos frutos de melancia estão associados a fatores genéticos, climáticos e fitotécnicos, sendo a nutrição da planta de fundamental importância para se conseguir resultados satisfatórios. Nesse viés, o nitrogênio e potássio são os nutrientes extraídos em maior quantidade pela planta de melancia (Grangeiro \& Cecílio Filho, 2004). De modo geral, pode-se considerar que o nitrogênio é o nutriente com maior efeito na produção, enquanto o potássio é o nutriente com maior efeito na qualidade dos frutos (Feltrim, 2010). Outro fator que interfere na quantidade e qualidade dos 
frutos é a densidade populacional, uma vez que variações no espaçamento entre plantas e/ou entre linhas podem diminuir ou aumentar a exigência nutricional da planta, em consequência altera o desenvolvimento desta e a resposta aos fatores de produção (Feltrim, 2010).

Para Rocha et al. (2020) a escassez de informações técnicas, principalmente aquelas relacionadas à nutrição de plantas, e que afeta diretamente na produtividade e qualidade dos frutos, são entraves ao crescimento da área cultivada com melancia. Dessa forma, o objetivo deste trabalho foi avaliar o efeito da aplicação de diferentes dosagens de nitrogênio sobre os componentes de qualidade de frutos da melancia cultivar "Granada" no município de Arinos, região norte de Minas Gerais.

\section{Metodologia}

O desenvolvimento do projeto (plantio, manejo, tratos culturais e colheita) foi conduzido no período de setembro a dezembro de 2018, em área de cerrado, na fazenda Extrema, situada no município de Arinos - MG. As coordenadas

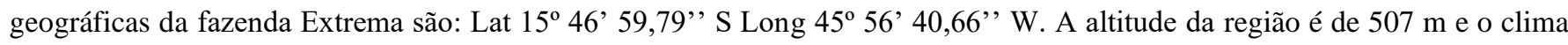
regional é do tipo Aw, segundo a classificação internacional de Köppen-Geiger (1936). O solo classificado como Latossolo, de textura franco-arenosa, apresentou os seguintes atributos químicos (Tabela 1), determinados pelos métodos descritos no Manual de Métodos de Análise de Solo (EMBRAPA, 1997).

Tabela 1. Resumo dos atributos químicos do solo da área experimental na fazenda Extrema, Arinos - MG.

\begin{tabular}{|c|c|c|c|c|c|c|c|c|c|}
\hline Camada & $\mathrm{pH}$ & $\mathrm{P}$ & K & $\mathrm{Ca}$ & $\mathrm{Mg}$ & $\mathrm{Al}$ & $\mathrm{H}+\mathrm{Al}$ & CTC & $\mathrm{V}$ \\
\hline$(\mathrm{cm})$ & $\left(\mathrm{CaCl}_{2}\right)$ & $\left(\mathrm{mg} \mathrm{dm}^{-3}\right)$ & & …. & $\ldots .(\mathrm{cn}$ & $\left.\mathrm{n}^{-3}\right)$. & & $\ldots$ & $(\%)$ \\
\hline $0-20$ & 5,8 & 53,92 & 0,37 & 4,14 & 1,45 & 0,01 & 3,5 & 5,97 & 62,7 \\
\hline
\end{tabular}

Fonte: Laboratório de análise de solos - Nativa.

O experimento foi conduzido em condições de campo, de setembro a dezembro de 2018. O delineamento experimental utilizado foi o de blocos casualizados, com cinco tratamentos e quatro repetições, totalizando 20 parcelas, estas que juntas mediram $10 \mathrm{~m}$ de largura por $25 \mathrm{~m}$ de comprimento. A parcela experimental foi constituída de 2 linhas de 5 metros de comprimento, com espaçamento de 2,5 m entre linhas e $1 \mathrm{~m}$ entre plantas. Entre cada bloco e cada parcela experimental, continha, respectivamente, uma e duas fileiras de plantas como bordadura. Circundando toda a área útil do experimento, havia quatro fileiras de plantas no mesmo espaçamento do experimento, a qual serviu como bordadura.

Os tratamentos foram compostos de cinco níveis de adubação nitrogenada. Os níveis de adubação nitrogenada (T1, T2, T3, T4 e T5), representam 0, 40, 80, que é a dose recomendada para a cultura (Filgueira et al., 1999), 160 e $320 \mathrm{~kg}$ de nitrogênio por hectare, que foram aplicados na forma de ureia ( $48 \%$ de $\mathrm{N}$ ), parcelados igualmente em três vezes, sendo aos 15 , 30 e 45 dias após a emergência (DAE).

A melancia foi semeada diretamente no campo, em covas, medindo $0,4 \times 0,4 \times 0,4 \mathrm{~m}$ de largura, comprimento e profundidade, respectivamente, colocando-se uma semente por cova. A cova foi feita com o auxílio de enxadão, após ter sido feito os sulcos de plantio, colocando-se $120 \mathrm{~g}$ de NPK (4-14-8) em cada cova. Aos 15 dias após o plantio (DAP), foi feito o transplantio das mudas onde não houve a germinação das sementes.

O sistema de irrigação utilizado foi o localizado por gotejamento com emissores espaçados $0,5 \mathrm{~m}$ e com vazão nominal de 4,5 $\mathrm{L} \mathrm{h}^{-1}$ a uma pressão de serviço de $147 \mathrm{kPa}$.

A primeira colheita dos frutos foi realizada aos 75 DAE e se estendeu até os $84 \mathrm{DAE}$, após terem sido identificados os parâmetros indicadores do ponto de colheita, como: mudança de coloração da parte do fruto em contato com o solo, secamento da gavinha mais próxima do fruto, mudança na casca do fruto que passa de verde brilhante para um tom mais opaco e o som 
“oco" ao bater no fruto com o nó do dedo.

As análises dos frutos foram feitas logo após a colheita, no laboratório de Química do Instituto Federal do Norte de Minas Gerais - Campus Arinos. Para isso, foram realizadas avaliações envolvendo os componentes de qualidade da melancia coletando dados referentes aos frutos representativos, provenientes da segunda colheita, escolhidos de forma aleatória e livres de deformidades como manchas, injúrias ou qualquer tipo de dano aparente.

Foram realizados testes físico-químicos para a determinação de Sólidos Solúveis Totais ( ${ }^{\circ}$ Brix), potencial hidrogeniônico (pH), Acidez Titulável e Espessura de Casca, da seguinte forma: SST - Sólidos Solúveis Totais: foram obtidos a partir do miolo da polpa, utilizando 3 a 4 gostas da amostra homogeneizada. A leitura feita por refratometria com correção da temperatura, conforme metodologia descrita pelo IAL (2008); AT - Acidez Titulável: foi obtida a partir da polpa, determinada por titulometria de neutralização, pela titulação de $10 \mathrm{~g}$ de polpa, homogeneizada e diluída para $100 \mathrm{~mL}$ em água destilada, com solução padronizada de $\mathrm{NaOH}$ a $0,1 \mathrm{~mol} \mathrm{~L}^{-1}$, com ponto de viragem no $\mathrm{pH} 8,2$, de conformidade com o método do IAL (2008); pH - Potencial Hidrogeniônico: foi obtido a partir da polpa, determinado em amostras constituídas de $10 \mathrm{~g}$ de polpa em $100 \mathrm{~mL}$ de água destilada, utilizando-se do pHmetro de bancada, marca MS Tecnopon modelo mPA210, com base no método do IAL (2008); Espessura da casca - pela medida da casca do fruto com o auxílio de paquímetro graduado manual.

Os dados coletados foram tabulados e, em seguida, submetidos à análise de variância e regressão. Os testes estatísticos para validação dos dados foram realizados com o auxílio do programa estatístico SISVAR versão 5.6 (Ferreira, 2014).

\section{Resultados e Discussão}

As dosagens de nitrogênio afetaram significativamente a qualidade química dos frutos, no que se refere aos conteúdos médios de Sólidos Solúveis Totais (SST), Acidez Titulável (AT) e a Espessura de Casca. O pH dos frutos não foi influenciado pelos tratamentos dentro dos percentuais estabelecidos de 1 ou $5 \%$ de probabilidade. Observaram-se valores médios de 8,21 ${ }^{\circ}$ Brix para SST, 1,48 g de ácido cítrico $100 \mathrm{~g}^{-1}$ de polpa para AT, 5,57 para pH e 11,77 mm para a espessura de casca (Tabela 2).

Tabela 2. Resumo da análise de variância de da melancia "Granada", tipo Crimson Sweet, submetidas a diferentes doses de adubação nitrogenada.

\begin{tabular}{lccc}
\hline Fonte de variação & Quadrados médios & Coeficiente de variação (\%) & Média Geral \\
\hline SST $\left(\mathrm{g}\right.$ de ácido cítrico $\left.100 \mathrm{~g}^{-1}\right)$ & $0,030^{*}$ & 1,53 & 8,21 \\
AT $\left({ }^{\circ}\right.$ Brix) & $0,058^{* *}$ & 4,05 & 1,48 \\
pH & $0,016^{\text {ns }}$ & 1,20 & 5,57 \\
ESP $(\mathrm{mm})$ & $1,355^{* *}$ & 3,61 & 11,77 \\
\hline
\end{tabular}

ns - não significativo, * - significativo a nível de 5\% de probabilidade, ** - significativo ao nível de 1\% de probabilidade pelo teste F, SST Sólidos Solúveis Totais, AT - Acidez Titulável, pH - Potencial Hidrogeniônico, ESP - Espessura de Casca. Fonte: Autores (2022).

Os parâmetros de qualidade dos frutos observados por Andrade Junior et al (2007), quando avaliaram frequência de aplicação de nitrogênio e de potássio por gotejamento em Parnaíba, PI, não foram influenciados significativamente pelos tratamentos. Mousinho (2003), em experimento no município de Fortaleza, CE, observou que a aplicação de N influenciou o SST dos frutos de melancia, reduzindo-os com o aumento do nível.

Os valores médios dos sólidos solúveis totais ou ( ${ }^{\circ}$ Brix), obtidos a partir da polpa dos frutos da melancia em função dos níveis de adubação nitrogenada estão expostos na Figura 1a. Foi possível ajustar um modelo quadrático significativo $(\mathrm{p}<0,05)$ em função das doses de nitrogênio, obtendo-se um valor máximo de $8,27{ }^{\circ} \mathrm{Brix}$, com $171,7 \mathrm{~kg} \mathrm{ha}^{-1}$ de $\mathrm{N}$, cujo 
resultado foi inferior ao observado por Araújo et al. (2011) que obtiveram valores máximos de 12,23 ${ }^{\circ}$ Brix, ao nível de 162,7 $\mathrm{kg} \mathrm{ha}^{-1} \mathrm{de} \mathrm{N}$, aplicado manualmente. Silva et al (2015), analisando a resposta da melancia à adubação nitrogenada e potássica, obtiveram valores semelhantes, visto que apresentaram média de $8,22^{\circ}$ Brix e valor máximo de $8,5{ }^{\circ}$ Brix na dose de $38,57 \mathrm{~kg}$ ha $^{-1}$ de N. Resultado contrário foi obtido por Andrade Junior et al. (2006), quando a aplicação de diferentes níveis de N, via fertirrigação, não influenciou os valores de SST.

Figura 1. Variação média de sólidos solúveis totais (A), acidez titulável (g de ácido cítrico $100 \mathrm{~g}^{-1}$ ) (B), espessura de casca (C) e pH (D) em função dos níveis de nitrogênio.
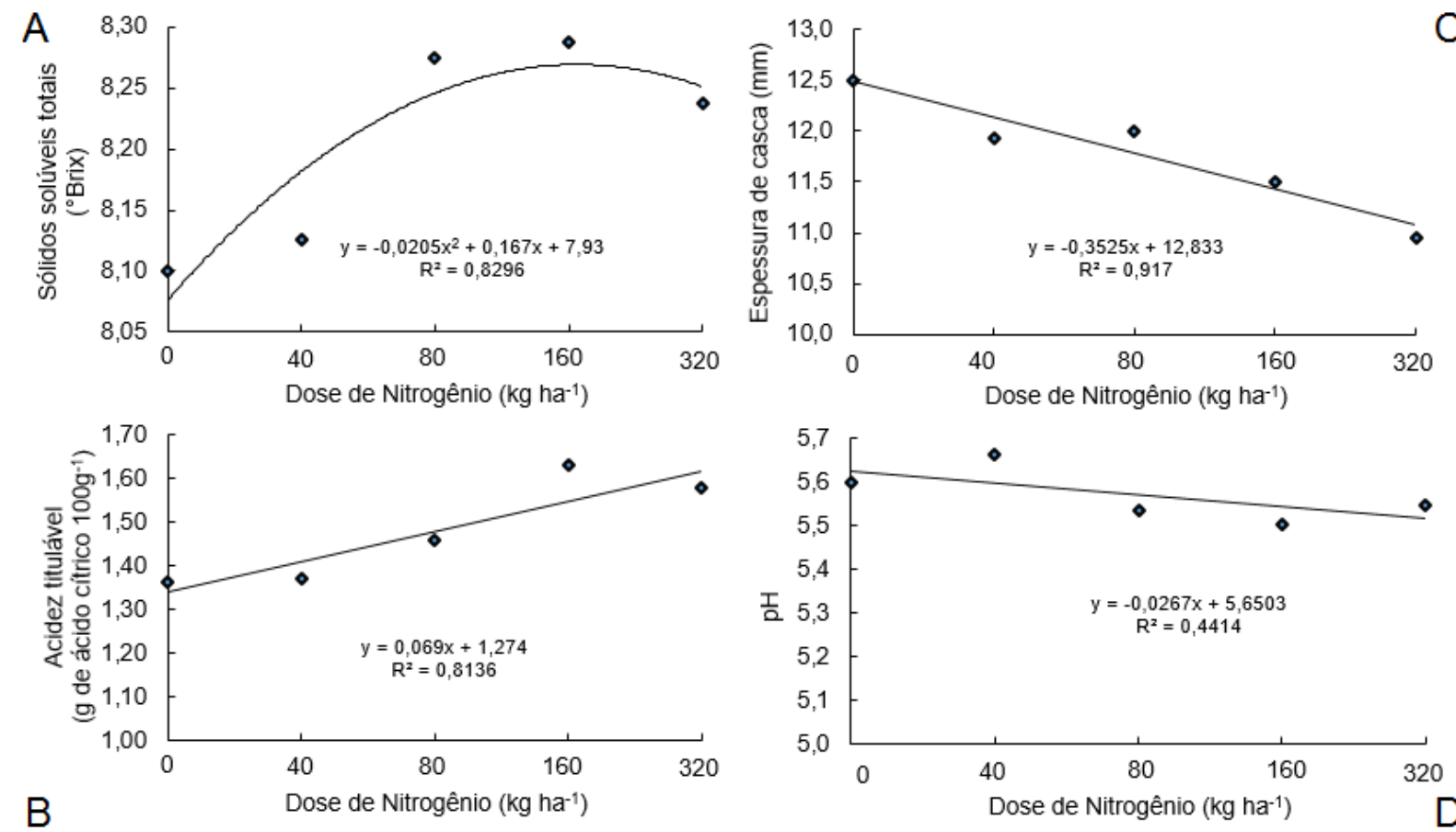

Fonte: Autores (2022).

Durante o período experimental, as variáveis climáticas foram limitantes ao desenvolvimento e à produção da cultura. As precipitações ocorreram durante o semeio, início da fase vegetativa e durante a colheita, interferindo na produção de flores e na qualidade dos frutos colhidos. Isso pode ser a causa dos baixos valores de sólidos solúveis encontrados neste trabalho. Weston e Barth (1997) e Fabeiro et al. (2002) verificaram que, sob condições de estresse hídrico, os frutos de tomate e melão tiveram seus níveis de sólidos solúveis totais aumentados. O mesmo foi evidenciado por Granjeiro e Cecílio Filho (2004), em experimento com melancia próximo à cidade de Borborema, SP, que obtiveram maiores teores de SST em período com baixa precipitação pluviométrica. Para Oliveira et al. (2019) os fatores ambientais, além dos fatores genéticos, influenciam a composição química dos vegetais, como a melancia, o que resulta na variação da composição química dos frutos, que influenciam diretamente no grau de maturação antes da colheita e nas condições de maturação pós-colheita e de armazenagem.

Altos teores de SST em frutos de melancia são bastante desejáveis e de grande aceitação, pois este índice é considerado parâmetro importante em muitos países, inclusive no Brasil (Bleinroth, 1994), visto que 8\% representa o mínimo aceitável para a comercialização.

Em relação à acidez titulável, foi ajustado um modelo linear crescente significativo a 1\% de probabilidade, obtendo-se um valor médio máximo de $1,63 \mathrm{~g}$ de ácido cítrico $100 \mathrm{~g}^{-1}$ de polpa ao nível de adubação de $160 \mathrm{~kg} \mathrm{ha}^{-1}$ de $\mathrm{N}$ (Figura $1 \mathrm{~b}$ ). $\mathrm{O}$ mesmo comportamento foi evidenciado por Medeiros et al. (2015), em experimento com plantas de cobertura e doses de N, atingindo um valor superior de 2,92 g de ácido cítrico $100 \mathrm{~g}^{-1}$ de polpa ao nível de $225 \mathrm{~kg} \mathrm{ha}^{-1}$ de N. Barros et al. (2012) 
obtiveram valores semelhantes, em que os autores constataram significância de 1\% em um modelo quadrático, com máxima de 1,67 g de ácido cítrico $100 \mathrm{~g}^{-1}$ de polpa ao nível de $155 \mathrm{~kg} \mathrm{ha}^{-1} \mathrm{de}$ N. Resultados contrários foram obtidos por Andrade Júnior et al. (2007), em que a acidez titulável não foi influenciada pelos tratamentos.

A acidez devida a ácidos orgânicos é uma característica importante no que se refere à palatabilidade de muitos frutos. Com poucas exceções, diminui com a maturação, em decorrência do processo respiratório ou de sua conversão em açúcares (Kader, 1978; Pretty, 1982).

Já a espessura da casca dos frutos foi diminuindo à medida que se aumentava a dose de nitrogênio (Figura 1c), seguindo um modelo linear significativo ( $\mathrm{p}<0,01$ ), até um mínimo de $10,9 \mathrm{~mm}$ ao nível de $320 \mathrm{~kg} \mathrm{ha}^{-1}$ de $\mathrm{N}$. Valores semelhantes foram observados por Ramos et al. (2009) e Granjeiro e Cecílio Filho (2004) em experimentos analisando a qualidade de frutos de melancia. Isso significa que, com o aumento das dosagens de nitrogênio, aumenta-se também a relação polpa/casca. Apesar de ser uma característica desejada, o afinamento em excesso da casca pode prejudicar a resistência do fruto ao transporte, do manejo ao acondicionamento, haja vista que, entre os cultivares utilizados no Brasil, aqueles de frutos com casca mais espessa são mais adequados ao tipo de transporte predominante no mercado interno (Tavares et al. 2019).

Não houve efeito significativo ( $>0,05)$ dos tratamentos sobre os valores do $\mathrm{pH}$ da polpa da melancia, apresentando uma média de 5,57 (Figura 1d). Resultado similar foi encontrado por Andrade Junior et al. (2006). Esses resultados estão de acordo com os observados por Morais et al. (2008), visto que não se observou influência da adubação nitrogenada no pH de frutos de melancia nos níveis de 75 a $300 \mathrm{~kg} \mathrm{ha}^{-1} \mathrm{de} \mathrm{N}$.

Os diferentes resultados encontrados na literatura podem ser atribuídos à forma de aplicação e fontes do adubo nitrogenado, a cultivar utilizada, ao manejo da irrigação e às condições edafoclimáticas onde os experimentos foram conduzidos.

\section{Considerações Finais}

Os componentes de qualidade dos frutos foram afetados significativamente pelas doses de nitrogênio, com exceção do pH. O maior teor de Sólidos Solúveis Totais, $8,27^{\circ}$ Brix, foi obtido ao nível de $171,7 \mathrm{~kg} \mathrm{ha}^{-1} \mathrm{de} \mathrm{N}$. A acidez titulável seguiu um modelo linear crescente ( $\mathrm{p}<0,01$ ), com valor médio máximo de $1,63 \mathrm{~g}$ de ácido cítrico $100 \mathrm{~g}^{-1}$ de polpa ao nível de $160 \mathrm{~kg}$ $\mathrm{ha}^{-1}$ de N. A espessura de casca dos frutos seguiu um modelo linear decrescente $(\mathrm{p}<0,01)$, reduzindo com o aumento da dose de N. Mais estudos são necessários visando relacionar componentes de qualidade dos frutos da melancia, com características vegetativas, como crescimento morfológico e suas interações ambientais.

\section{Referências}

Andrade Júnior, A. S., Dias, N. D. S., de lira, R. B., Figueredo Júnior, L. G. M., \& Daniel, R. (2007). Frequência de aplicação de nitrogênio e de potássio via água de irrigação por gotejamento na cultura da melancia em Parnaíba, PI. Agropecuária Cientifica no Semiárido (ACSA) 03, 01-07.

Andrade Júnior, A. S. D., Dias, N. D. S., Figueiredo Junior, L. G., Ribeiro, V. Q., \& Sampaio, D. B. (2006). Produção e qualidade de frutos de melancia à aplicação de nitrogênio via fertirrigação. Revista Brasileira de Engenharia Agrícola e Ambiental, 10, 836-841.

Bleinroth, E. W. (1994). Determinação do ponto de colheita. In: Gorgatti Neto, A., Gayet, J. P., Bleinroth, E. W., Matallo, M., Garcia, E. E. C., Garcia, A. E., \& Bordin, M. R. Melão para exportação: procedimentos de colheita e pós-colheita. Brasília: EMBRAPA-SPI (Série Publicações Técnicas), p.11-21.

Brasil, Instituto Brasileiro de Geografia e Estatística [IBGE]. (2007). Produção Agrícola Nacional: Lavouras Temporárias. https://cidades.ibge.gov.br/brasil/panorama.

Brasil, Instituto Brasileiro de Geografia e Estatística [IBGE]. (2012). Produção Agrícola Nacional: Lavouras Temporárias. http://www.sidra.ibge.gov.br

Conab - Companhia Nacional de Abastecimento. Boletim Hortigranjeiro. (2018). 4 (2), https://www.conab.gov.br/info-agro/hortigranjeiros-prohort/boletimhortigranjeiro

Fabeiro, C., Olalla, F. M. de Santa, Juan, J. A. Fabeiro, C., de Santa Olalla, F. M., \& De Juan, J. A. (2002). Production of muskmelon (Cucumis melo L.) under controlled deficit irrigation in a semi-arid climate. Agricultural water management, 54(2), 93-105. 
Feltrim, A. L. (2010). Produtividade de melancia em função da adubação nitrogenada, potássica e população de plantas. (2010). 87 f. [Doctoral dissertation, Tese (Doutorado em Produção Vegetal) -Universidade Estadual Paulista, Jaboticabal].

Ferreira, D. F. (2014). Sisvar: A Guide for its Bootstrap procedures in multiple comparisons. Ciência e Agrotecnologia, 38 (2), 109-112. http://dx.doi.org/10.1590/S1413-70542014000200001

Ferreira, M. A. J., Queiróz, M. A. D., Braz, L. T., \& Vencovsky, R. (2003). Correlações genotípicas, fenotípicas e de ambiente entre dez caracteres de melancia e suas implicações para o melhoramento genético. Horticultura Brasileira, 21 (2), 438-4442.

Filgueira, F. A. R., Carrijo, I.V., \& Avelar Filho, J. A. Melancia. (1999). In: Ribeiro, A. C., Guimarães, P. T. G. \& Alvarez V., Vitor. H., (eds.) Recomendações para o uso de corretivos e fertilizantes em Minas Gerais: $5^{\text {a }}$ Aproximação. Comissão de Fertilidade do Solo do Estado de Minas Gerais, Viçosa - MG, 360 .

Grangeiro, L. C. \& Cecílio Filho, A. B. (2004). Qualidade de frutos de melancia em função de fontes e doses de potássio. Horticultura Brasileira, 22 (3), 647650 .

Cecílio Filho, A. B., \& Grangeiro, L. C. (2004). Qualidade de frutos de melancia sem sementes em função de fontes e doses de potássio. Ciência $e$ Agrotecnologia, 28 (3), 570-576.

Hexem, R. W., \& Heady, E. O. (1978). Water production functions for irrigated agriculture. The Iowa University Press, 215.

Kader A. A. (1978). Quality factors: definition and evaluation for fresh horticultural crops. In: KADER A. A. Postharvest Technology of Horticultural Crops. California: University of California, 118-121.

Köppen, W \& Geiger, R. (1936). Das Geographisca System der Klimate. In: W. Koppen and G. C. Geiger, Eds., Handbuch der Klimatologie, Gebr, Borntraeger, 44.

Medeiros, R. D., da Silva, E. S., Carmo, I. D. S., Monteiro Neto, J. L. L., do Nascimento, F. R., \& da Silva, A. P. (2015). Qualidade de frutos de melancia sob plantas de cobertura e doses de nitrogênio. In: Congresso Brasileiro De Ciência Do Solo, (anais de congresso). Natal: Sociedade Brasileira de Ciência do Solo.

Miguel, A. C. A., Dias, J. R. P. S., \& Spoto, M. H. F. (2007). Efeito do cloreto de cálcio na qualidade de melancias minimamente processadas. Horticultura Brasileira, 25 (3), 442-446.

Morais, N. B., Bezerra, F. M. L., de Medeiros, J. F., \& Chaves, S. W. P. (2008). Resposta de plantas de melancia cultivadas sob diferentes níveis de água e de nitrogênio. Revista Ciência Agronômica, 39(3), 369-377.

Mousinho, F. E. P., Costa, R. N. T., de Souza, F., \& Gomes Filho, R. R. (2003). Função de resposta da melancia à aplicação de água e nitrogênio para as condições edafoclimáticas de Fortaleza, CE. Irriga, 8(3), 264-272.

Oliveira, M. M. T., Alves, R. E., Silva, L. R., \& Aragão, A. S. (2019). Qualidade de frutos de híbridos de melancia com sementes. Revista de la Facultad de Agronomía, 118(1), 77-83.

Pretty, K. M. (1982). O potássio e a qualidade da produção agrícola. Potássio na agricultura brasileira. Piracicaba: Instituto da Potassa \& Fosfato: Instituto Internacional da Potassa, 177-194.

Ramos, A. R., Dias, R. D. C. S., \& Aragão, C. A. (2009). Densidades de plantio na produtividade e qualidade de frutos de melancia. Horticultura Brasileira, 27 (4), 560-564.

Rocha, P. H. F., Barbacena, D. R., França, L. C., Gonçalves, R. C., Nascimento, C. A., Leal, F., \& Adorian, G. C. (2020). Incremento de matéria seca e marcha de acúmulo de nutrientes em melancia cv. Top Gun em área de várzea no Tocantins. Revista Agri-Environmental Sciences, 6(e020006), 1-10.

Tavares, A. T., Lopes, D. A. P. S., Alves, F. Q. G., Santos, G. R., \& Nascimento, I. R. (2019). Heterose em híbridos de melancia. Revista de Agricultura Neotropical, 6(2), 26-33.

Weston, L. A., \& Barth, M. M. (1997). Preharvest factors affecting postharvest quality of vegetables. HortScience, 32 (5), 812-816. 